\title{
NON-FORMALITY OF THE ODD DIMENSIONAL FRAMED LITTLE BALLS OPERADS
}

\author{
SYUNJI MORIYA
}

\begin{abstract}
We prove that the chain operad of the framed little balls (or disks) operad is not formal as a non-symmetric operad over the rationals if the dimension of their balls is odd and greater than 4 .
\end{abstract}

\section{INTRODUCTION}

A chain operad $\mathcal{O}$ is said to be formal if $\mathcal{O}$ and the homology operad $H_{*}(\mathcal{O})$ is connected by a chain of weak equivalences (see section 2 for the precise definition).

The formality of the chain little balls operads is a very important property. It is first discovered by D. Tamarkin in the 2 dimensional case, and applied to the proof of deformation quantization, and generalized by M. Kontsevich to the arbitrary dimension ( see 23 for a detailed description of Kontsevich's sketchy construction). Combined with Goodwillie-Klein-Weiss embedding calculus, the formality is also used to compute the homology of knot spaces and more generally, embedding spaces (see [13, 15, 21, 24, 28]).

The framed little balls operads are cousins of the little balls operads, which encode rotations of balls, and appear in many areas. For example, They play an important role in the embedding calculus, in particular in the case that involving manifolds are not parallelizable (see [18, 19]).

J. Giansiracusa and P. Salvatore [17, Theorem.A] proved the formality of framed little 2-balls (disks) operad, and they questioned whether the framed little balls operads of higher dimensions are formal.

In this paper, we give an answer to this question for odd dimensions greater than 4 .

Theorem 1.1. Let $d$ be an odd number greater than 4. If the coefficients are the rational numbers, the d-dimensional chain framed little balls operad is not formal as a nonsymmetric operad.

It is clear that non-formality as a non-symmetric operad implies non-formality as a symmetric operad. Theorem 1.1 might be an unhappy result in view of computations on embedding spaces but the author expects the obstruction to formality given in section 4 will be useful to understand the higher information of the chain framed little disks operad.

We shall give an outline of the proof of Theorem 1.1. (We actually prove Theorem 2.3. part 1 of which is equivalent to Theorem 1.1.) We use the framed Kontsevich operad instead of the framed little balls operad since it has a structure of a multiplicative operad. To a multiplicative operad, J. E. McClure and J. H. Smith associated a cosimplical space. We associate a homology spectral sequence to the cosimplicial space by the BousfieldKan's procedure. In section 3. We see that the spectral sequences associated to the odddimensional framed Kontsevich operads do not degenerate at $E^{2}$-page. Here we actually deal with simpler operads, the framed choose-two operads, mainly. The non-degeneracy of the spectral sequences of the framed Kontsevich operads follows from that of the framed choose-two operads. We detect an element whose differential is non zero. The argument

Date: October 9, 2018.

The author is partially supported by JSPS KAKENHI Grant Number 26800037. 
is essentially a simple observation for the number of generators and degrees. Note that formality as a multiplicative operad implies degeneracy of the spectral sequence at $E^{2}$-page as in [15, 27, 22, so in turn, non-degeneracy of the spectral sequence implies non-formality as a multiplicative operad. But it is weaker than non-formality as a non-symmetric operad. For example, the 2-dimensional chain Kontsevich operad is not formal as a multiplicative operad though it is formal as (non-)symmetric operad (see 25]). To obtain the latter non-formality, we introduce an obstruction to formality which can be defined for any (possibly non-multiplicative) operad the homology of which is isomorphic to that of the framed little balls operad. This obstruction is something like "Massey triple bracket" for Gerstenhaber bracket (see Remark 4.3). Unlike the usual Massey triple product, our obstruction might depend not only on classes but also on cycles. (At least, we do not prove such independence.) To deal with technical issues about choices of cycles, we make use of a model category of operads. We prove the main theorem 1.1 (or 2.3) by using the fact that the obstruction is equal to the non-trivial differential of the spectral sequences for the framed operads.

Other non-formality results are found in [25, 26]. In [25] the authors proved nonformality of codimension one inclusion between the little balls operads. In [26], the author proved non-formality of Swiss-cheese operad and considered a general Massey product for partial compositions of operads.

\section{Preliminary}

In this section, we shall recall basic definitions and known results, and fix notations.

- In the present paper, the coefficients of all modules are in the field of rational numbers $\mathbb{Q}$. In particular, all homology groups are supposed to have the rational coefficients.

- The term "operad" means non-symmetric operad. A (non-symmetric) operad in a symmetric monoidal category $(\mathcal{C}, \otimes, 1)$ consists of a sequence of objects $\mathcal{O}(0)$, $\mathcal{O}(1), \ldots, \mathcal{O}(n), \ldots$ in $\mathcal{C}$ and a set of partial compositions $-\circ_{i}-: \mathcal{O}(m) \otimes \mathcal{O}(n) \rightarrow$ $\mathcal{O}(m+n-1)$. which satisfies axioms of associativity and unity (see [16, section 2] or [4, Variants 1.2]). A morphism of operads $f: \mathcal{O} \rightarrow \mathcal{P}$ is a sequence of morphisms $\left\{f_{n}: \mathcal{O}(n) \rightarrow \mathcal{P}(n)\right\}_{n \geq 0}$ which is compatible with the partial compositions and preserves the unit. We call $\mathcal{O}(n)$ the part of arity $n$ of $\mathcal{O}$. If $\mathcal{C}$ is the monoidal category Top of topological spaces, continuous maps, and the cartesian product (resp. $\mathcal{C H}$ of chain complex, chain maps, and the tensor product over $\mathbb{Q}$ ), we call an operad in $\mathcal{C}$ a topological operad (resp. a chain operad). The singular chain functor $C_{*}: T o p \rightarrow \mathcal{C H}$ (with the rational coefficients) assigns a chain operad to a topological operad.

- A morphism $f: \mathcal{O} \rightarrow \mathcal{P}$ of topological operads (resp. chain operads) is said to be a weak equivalence if $f_{n}: \mathcal{O}(n) \rightarrow \mathcal{P}(n)$ is a weak homotopy equivalence (resp. a quasi-isomorphism ) for each $n \geq 0$. A chain operad $\mathcal{O}$ is said to be formal if there exists a chain of weak equivalences of operads connecting $\mathcal{O}$ and $H_{*}(\mathcal{O})$ :

$$
\mathcal{O} \longleftarrow \mathcal{O}_{1} \longrightarrow \cdots \longleftarrow \mathcal{O}_{N} \longrightarrow H_{*}(\mathcal{O}),
$$

where $H_{*}(\mathcal{O})$ is a chain operad given by $H_{*}(\mathcal{O})(n)=H_{*}(\mathcal{O}(n))$ with the zero differential.

- We shall deal with the Kontsevich operad $\mathcal{K}_{d}$ and the choose-two operad $\mathcal{S}_{d}$ (see [13, section 3, 4, Example 7.4], where the term "choose-two operad" represents a different concept, in their notation, our $\mathcal{S}_{d}$ is equal to $\left(S^{d-1}\right)^{S_{\bullet}^{2}}$, or [12, section 2], where the choose-two operad is denoted by $B_{n}$ ). We shall recall the definition of operads $\mathcal{K}_{d}$ and $\mathcal{S}_{d}$ in some details. Let $d$ be a positive integer and $S^{d-1}=\{x \in$ $\left.\mathbb{R}^{d} \mid\|x\|=1\right\}$ be the standard unit $(d-1)$-sphere in the Euclidean space $\mathbb{R}^{d}$. We 
put

$$
\mathcal{S}_{d}(n)=\prod_{1 \leq i<j \leq n} S^{d-1}
$$

Let $F_{n}\left(\mathbb{R}^{d}\right)$ denote the space of ordered configurations of $n$-points in $\mathbb{R}^{d}$. We define a map $\theta: F_{n}\left(\mathbb{R}^{d}\right) \rightarrow \mathcal{S}_{d}(n)$ by

$$
\theta\left(x_{1}, \ldots, x_{n}\right)=\left(\frac{x_{j}-x_{i}}{\left|x_{j}-x_{i}\right|}\right)_{i, j}
$$

In other words, the $(i, j)$-component of $\theta\left(x_{1}, \ldots, x_{n}\right)$ is the direction vector from $x_{i}$ to $x_{j}$. The space $\mathcal{K}_{d}(n)$ is defined to be the closure of the image of $\theta$ in $\mathcal{S}_{d}(n)$. We set $\mathcal{S}_{d}(n)=\mathcal{K}_{d}(n)=*$ for $n=0,1$. To get the partial composition of $\mathcal{S}_{d}$, we shall define a map

$$
\left(-\circ_{i}-\right): F_{m}\left(\mathbb{R}^{d}\right) \times F_{n}\left(\mathbb{R}^{d}\right) \rightarrow \mathcal{S}_{d}(m+n-1)
$$

for $i=1, \ldots, m$. Take $\left(x_{1}, \ldots, x_{m}\right) \in F_{m}\left(\mathbb{R}^{d}\right)$ and $\left(y_{1}, \ldots, y_{n}\right) \in F_{n}\left(\mathbb{R}^{d}\right)$. Intuitively speaking, $\left(x_{1}, \ldots, x_{m}\right) \circ_{i}\left(y_{1}, \ldots, y_{n}\right)$ is represented by the configuration made from $x_{1}, \ldots, x_{m}$ by replacing $x_{i}$ with $y_{1}, \ldots, y_{n}$ which are infinitesimally rescaled. More precisely, we first put

$$
\left(w_{1}^{r}, \ldots, w_{m+n-1}^{r}\right)=\left(x_{1}, \ldots, x_{i-1}, x_{i}+r y_{1}, \ldots x_{i}+r y_{n}, x_{i+1}, \ldots, x_{n}\right)
$$

where $r y_{k}$ means scalar multiplication by a positive number $r$. Note that if $r$ is sufficiently small, $\left(w_{1}^{r}, \ldots, w_{m+n-1}^{r}\right)$ belongs to $F_{m+n-1}\left(\mathbb{R}^{d}\right)$. Then, we set

$$
\left(x_{1}, \ldots, x_{m}\right) \circ_{i}\left(y_{1}, \ldots y_{n}\right)=\lim _{r \rightarrow 0} \theta\left(w_{1}^{r}, \ldots, w_{m+n-1}^{r}\right) .
$$

Note that the limits of direction vectors of $w_{1}^{r}, \ldots w_{m+n-1}^{r}$ depend only on the direction vectors between two of $x_{1}, \ldots, x_{m}$ and of $y_{1}, \ldots, y_{n}$, so the above map $\left(-\circ_{i}-\right)$ is naturally extended to a map $\left(-\circ_{i}-\right): \mathcal{S}_{d}(m) \times \mathcal{S}_{d}(n) \longrightarrow \mathcal{S}_{d}(m+$ $n-1)$, which gives $\mathcal{S}_{d}$ a structure of an operad (see [12, section 2] for an explicit formula of this partial composition). It is known that the restriction of this partial composition to $\mathcal{K}_{d}$ factors through $\mathcal{K}_{d}$ and we endow $K_{d}$ this structure of a suboperad of $\mathcal{S}_{d}$. (see [13, Theorem 4.5]). $\mathcal{K}_{d}$ and the little balls operad $\mathcal{D}_{d}$ is known to be weak equivalent as topological operads for each $d \geq 1$. In other words, $\mathcal{K}_{d}$ and $\mathcal{D}_{d}$ are connected by a chain of weak equivalences.

- The framed version of $\mathcal{K}_{d}$ and $\mathcal{S}_{d}$ is given as a semidirect product with the rotation group $S O_{d}$. A notion of a semidirect product of an operad is introduced by N. Wahl and P. Salvatore [8, Definition 2.1]. Let $\mathcal{O}$ be a topological operad and $G$ be a topological group. Suppose each $\mathcal{O}(n)$ has a $G$-action which satisfies some compatibility conditions (see ibid.). We define a topological operad $\mathcal{O} \rtimes G$ by

$$
\begin{aligned}
\mathcal{O} \rtimes G(n) & =\mathcal{O}(n) \times G^{n} \\
\left(x ; g_{1}, \ldots, g_{m}\right) \circ_{i}\left(y ; h_{1}, \ldots, h_{n}\right) & =\left(x \circ_{i}\left(g_{i} \cdot y\right) ; g_{1}, \ldots, g_{i} h_{1}, \ldots, g_{i} h_{n}, g_{i+1}, \ldots, g_{m}\right)
\end{aligned}
$$

for $\left(x, g_{1}, \ldots, g_{m}\right) \in \mathcal{O} \rtimes G(m),\left(y, h_{1}, \ldots, h_{n}\right) \in \mathcal{O} \rtimes G(n)$. The most important example of semi-direct products is the d-dimensional framed little balls operad which is the semi-direct product with respect to the natural action of the rotation group $S O_{d}$ on the $d$-dimensional little balls operad $\mathcal{D}_{d}$ (see Example 2.2 of ibid.). $\mathcal{K}_{d}$ and $\mathcal{S}_{d}$ also have the actions of $S O_{d}$ which are induced by the restriction of the natural action on $\mathbb{R}^{d}$ to $S^{d-1}$. The inclusion $\mathcal{K}_{d} \rightarrow \mathcal{S}_{d}$ preserves these actions. For $\mathcal{O}=\mathcal{D}_{d}, \mathcal{K}_{d}$, or $\mathcal{S}_{d}$, we put $f \mathcal{O}=\mathcal{O} \rtimes S O_{d}$. We call $f \mathcal{K}_{d}$ (resp. $f \mathcal{S}_{d}$ ) the $d$-dimensional framed Kontsevich operad (resp. the d-dimensional framed choosetwo operad). $f \mathcal{K}_{d}$ and the framed little balls operad $f \mathcal{D}_{d}$ are known to be weak equivalent as topological operads (see [12, section 3]). 
- We will use McClure-Smith's procedure which produces cosimplicial objects $\mathcal{O}^{\bullet}$ from a multiplicative operad (see [10]). Let $\mathcal{A}$ denote the associative operad. (We consider the unital version so that $\mathcal{A}(0)$ is a point). A multiplicative operad (or an operad with multiplication) is a morphism from $\mathcal{A}$ to an operad $\mathcal{O}$ and a morphism of multiplicative operads is the same as a morphism of the under category. We can associate a cosimplicial space $\mathcal{O}^{\bullet}$ to a multiplicative operad $f: \mathcal{A} \rightarrow \mathcal{O}$ as follows. Let $\mu \in \mathcal{O}(2)$ (resp. $e \in \mathcal{O}(0)$ ) be the image of the unique element of $\mathcal{A}(2)$ (resp. of $\mathcal{A}(0)$ ) by $f$. We put $\mathcal{O}^{n}=\mathcal{O}(n)$ for each integer $n \geq 0$, and we define the coface $d^{i}: \mathcal{O}^{n} \rightarrow \mathcal{O}^{n+1}$ and codegeneracy $s^{i}: \mathcal{O}^{n} \rightarrow \mathcal{O}^{n-1}$ by

$$
\begin{aligned}
d^{0}(x)=\mu \circ_{2} x, d^{n+1}(x) & =\mu \circ_{1} x, d^{i}(x)=x \circ_{i} \mu \quad(1 \leq i \leq n) \\
s^{i}(x) & =x \circ_{i} e \quad(1 \leq i \leq n)
\end{aligned}
$$

The main advantage of the Kontsevich operad to the little balls operad is that it has a structure of mulitiplicative operad. We set $v_{0}=(1,0, \ldots, 0)$ as the base point of $S^{d-1}$. The map $\mathcal{A}(2) \rightarrow \mathcal{S}_{d}(2)=S^{d-1}$ taking the value on $v_{0}$ uniquely extends to a morphism $\mathcal{A} \rightarrow \mathcal{S}_{d}$. This morphism factors through $\mathcal{K}_{d}$. We regard $\mathcal{S}_{d}$ and $\mathcal{K}_{d}$ as multiplicative operads with these morphisms (see [12, section2], [13, Proposition 4.7] ). For $\mathcal{O}=\mathcal{S}_{d}, \mathcal{K}_{d}$, We also regard $f \mathcal{O}$ as a multiplicative operad with the composition $\mathcal{A} \rightarrow \mathcal{O} \subset f \mathcal{O}$ where the inclusion is induced by the inclusion to the unit $* \rightarrow S O(d)$. We deal with the cosimplicial objects $\mathcal{S}_{d}^{\bullet}, \mathcal{K}_{d}^{\bullet}$, $f \mathcal{S}_{d}^{\bullet}$, and $f \mathcal{K}_{d}^{\bullet}$.

- For a cosimplicial space $Y^{\bullet}$ let $E_{p, q}^{r}\left(Y^{\bullet}\right)$ denote the $E^{r}$-page of Bousfield-Kan spectral sequence associated to the cosimplicial chain complex $C_{*}\left(Y^{\bullet}\right)$ (Note that $E_{p, q}^{r}$ is the part of cosimplicial degree $-p$, chain degree $\left.q\right)$.

- For a topological multiplicative operad $\mathcal{O}, H H_{*, *}\left(H_{*}(\mathcal{O})\right)$ denotes the Hochschild homology of $H_{*}(\mathcal{O})$ considered as a chain operad with the zero differential. It is the homology group of the normalization of the cosimplical graded group $H_{*}(\mathcal{O})$ (see [11, section 3],[12, Definition 18], [13, Definition 2.17]). The Hochschild homology has the bigrading analogous to that of $E_{*, *}^{r}\left(Y^{\bullet}\right)$ and a natural structure of a Gerstenhaber alegbra. (see [3, section 2] where a Gerstenhaber algebra is called a G-algebra, and [11, section 3], [14, subsection 4.3]). $E_{*, *}^{2}\left(\mathcal{O}^{\bullet}\right)$ also has a structure of a Gerstenhaber algebra as it is naturally isomorphic to $H H_{*, *}\left(H_{*}(\mathcal{O})\right)$

- For $k=d, d-1, \omega \mathrm{SO}_{k}$ denotes the cobar complex for the coalgebra $H_{*}\left(S O_{k}\right)$ with Alexander-Whitney diagonal. $H_{*, *}\left(\omega \mathrm{SO}_{k}\right)$ denotes the total homology with the natural bigrading.

- For a pointed topological space $X$, let $\underline{X}^{\bullet}$ denote the usual cobar cosimplicial space (so $\underline{X}^{n}=X^{\times n}$ ), and $\Omega(X)$ denote the based loop space of $X$. We let Tot denote the homotopy totalization (or limit) functor for cosimplicial spaces.

Lemma 2.1 (Corollary 10 and Proposition 16 of [12]). For any integer $d \geq 2$, we have weak homotopy equivalences:

$$
\widetilde{\operatorname{Tot}}\left(\mathcal{S}_{d}^{\bullet}\right) \simeq \Omega^{2}\left(S^{d-1}\right), \quad \widetilde{\operatorname{Tot}}\left(f \mathcal{S}_{d}^{\bullet}\right) \simeq \Omega\left(S O_{d-1}\right) .
$$

The second equivalence is induced by the cosimplicial map $\mathrm{SO}_{d-1}{ }^{\bullet} \rightarrow f \mathcal{S}_{d}^{\bullet}$ defined by the usual inclusion $S O_{d-1} \rightarrow S O_{d}$ to the subgroup consisting of matrices whose first column is $v_{0}$, and the configurations which are the images by the fixed morphism $\mathcal{A} \rightarrow f \mathcal{S}_{d}$.

The following lemma is well-known. We denote by $\Omega\left(S O_{k}\right)_{1}$ the component of the based loop space of $\Omega\left(S O_{k}\right)$ containing constant loops for $k=d, d-1$.

Lemma 2.2. Let $d=2 m+1$ be an odd number greater than 2 .

(1) There exist isomorphisms of rational homology algebras:

$$
H_{*}\left(S O_{d}\right) \cong \bigwedge\left(\beta_{1}, \ldots, \beta_{m}\right), \quad H_{*}\left(S O_{d-1}\right) \cong \bigwedge\left(\beta_{1}, \ldots, \beta_{m-1}, e\right)
$$


Here, $\bigwedge$ denotes the free anti-commutative algebra, and we set $\operatorname{deg} \beta_{i}=4 i-1, \operatorname{deg} e=$ $2 m-1$. Furthermore, the generators can be taken as primitive elements with respect to the Alexander-Whitney diagonal.

(2) There exist isomorphisms of rational homology algebras:

$$
H_{*}\left(\Omega\left(S O_{d}\right)_{1}\right) \cong \mathbb{Q}\left[\gamma_{1}, \ldots, \gamma_{m}\right], \quad H_{*}\left(\Omega\left(S O_{d-1}\right)_{1}\right) \cong \mathbb{Q}\left[\gamma_{1}, \ldots, \gamma_{m-1}, f\right]
$$

Here, $\mathbb{Q}[\cdots]$ denotes the free commutative algebra, and we set $\operatorname{deg} \gamma_{i}=4 i-2, \operatorname{deg} f=$ $2 m-2$.

As the framed little balls operads are weak equivalent to the framed Kontsevich operads, Theorem 1.1 is equivalent to the part 1 of the following theorem which we will prove in the rest of the paper.

Theorem 2.3. Let $d$ be an odd number greater than 4 .

(1) The chain operad $C_{*}\left(f \mathcal{K}_{d}\right)$ of the framed d-dimensional Kontsevich operad is not formal over $\mathbb{Q}$.

(2) The chain operad $C_{*}\left(f \mathcal{S}_{d}\right)$ of the framed d-dimensional choose-two operad is not formal over $\mathbb{Q}$.

\section{Non-DEGEnERACY OF THE SPECTRAL SEQUENCES ASSOCIATED TO THE FRAMED} OPERADS

In the rest of paper, $d=2 m+1$ denotes an odd number greater than 4 .

Lemma 3.1. Let $\mathcal{O}$ be $\mathcal{K}_{d}$ or $\mathcal{S}_{d}$.

(1) There exists an isomorphism of algebras

$$
E_{p, q}^{2}\left(f \mathcal{O}^{\bullet}\right) \cong \bigoplus_{p_{1}+p_{2}=p, q_{1}+q_{2}=q} H H_{p_{1}, q_{1}}\left(H_{*}(\mathcal{O})\right) \otimes H_{p_{2}, q_{2}}\left(\omega \mathrm{SO}_{d}\right)
$$

which is natural with respect to the inclusion $i: \mathcal{K}_{d} \rightarrow \mathcal{S}_{d}$, and

(2) Let $\bar{\beta}_{m}$ be the image of the element $\beta_{m}$ in Lemma 2.2 by the natural map $H_{4 m-1}\left(S O_{d}\right) \rightarrow H_{-1,4 m-1}\left(\omega \mathrm{SO}_{d}\right)$. When we regard $1 \otimes \bar{\beta}_{m}$ as an element of $E^{2}\left(f \mathcal{O}^{\bullet}\right)$ under the isomorphism of $(1), d^{2}\left(1 \otimes \bar{\beta}_{m}\right)$ is non-zero in $E^{2}\left(f \mathcal{O}^{\bullet}\right)$.

Proof. We shall prove the part 1. There is a natural isomorphism $E_{*, *}^{2}\left(f \mathcal{O}^{\bullet}\right) \cong$ $H H_{*, *}\left(H_{*}(f \mathcal{O})\right)$. The action of the Hopf algebra $H_{*}\left(S O_{d}\right)$ on $H_{*}(\mathcal{O}(n))$ is trivial by the degree reason for any odd $d$, see [8, Theorem 5.4], so the semidirect product splits on the homology level (this is the point where we need $d$ is odd). It follows that as a cosimplical graded vector space, $H_{*}\left(f \mathcal{O}^{\bullet}\right)$ is isomorphic to the cosimplicial-levelwise tensor product of $H_{*}\left(\mathcal{O}^{\bullet}\right)$ and the cosimplicial cobar complex associated to $H_{*}\left(S O_{d}\right)$. This implies $H H_{*, *}\left(H_{*}(f \mathcal{O})\right) \cong H H_{*, *}\left(H_{*}(\mathcal{O})\right) \otimes H_{*, *}\left(\omega \mathrm{SO}_{d}\right)$.

We shall show the part 2 for $\mathcal{O}=\mathcal{S}_{d}$. In view of part 1 , we easily see $E_{p, q}^{2}\left(f \mathcal{S}_{d}^{\bullet}\right)=0$ for $q<-\frac{d-1}{2} p$. As $d \geq 5$, we have the inequality $\frac{d-1}{2}>1$. Also, note that $H_{*}\left(\Omega\left(\left(S O_{d-1}\right)_{\mathbb{Q}}\right)\right) \cong H_{*}\left(\Omega\left(S O_{d-1}\right)_{1}\right)$ as $\pi_{1}\left(S O_{d-1}\right) \otimes \mathbb{Q}=0$, where $(-)_{\mathbb{Q}}$ denotes the rationalization. These facts and [1, Theorem 3.4] imply $E_{*, *}^{r}\left(f \mathcal{S}_{d}^{\bullet}\right)$ converges to $H_{*}\left(\Omega\left(S O_{d-1}\right)_{1}\right)$. Note that the partial compositions of $\mathcal{S}_{d}$ is defined by the diagonal map of $S^{d-1}$. This fact and the formality of the rational singular chain coalgebra $C_{*}\left(S^{d-1}\right)$ imply the formality of the rational singular chain multiplicative operad $C_{*}\left(\mathcal{S}_{d}\right)$. This multiplicative operad formality implies the Bousfield-Kan spectral sequence associated to the cosimplicial space $\mathcal{S}_{d}^{\bullet}$ degenerates at $E^{2}$-page, see the proof of Theorem 1.4 in 27 . This spectral sequence converges to the homology $H_{*}\left(\widetilde{\operatorname{Tot}}\left(\mathcal{S}_{d}^{\bullet}\right)\right)$. By these observations and Lemma 2.1] we have an isomorphism

$$
H H_{*, *}\left(H_{*}\left(\mathcal{S}_{d}\right)\right) \cong H_{*}\left(\Omega^{2} S^{d-1}\right) .
$$


Moreover, this isomorphism is an isomorphism of Gerstenhaber algebras. On the other hand, the following isomorphism of graded algebras is well-known.

$$
H_{*, *}\left(\omega \mathrm{SO}_{d}\right) \cong H_{*}\left(\Omega\left(S O_{d}\right)_{1}\right)
$$

Putting the isomorphisms (11), (21) and (3) into together, we obtain the following:

$$
E^{2}\left(f \mathcal{S}_{d}^{\bullet}\right) \cong H_{*}\left(\Omega^{2} S^{d-1}\right) \otimes H_{*}\left(\Omega\left(S O_{d}\right)_{1}\right) \Longrightarrow H_{*}\left(\Omega\left(S O_{d-1}\right)_{1}\right)
$$

Let $\{-,-\}$ denote the Gerstenhaber bracket (Brawder operation) on $H_{*}\left(\Omega^{2} S^{d-1}\right)$. It is well-known that $H_{*}\left(\Omega^{2} S^{d-1}\right)$ is generated by two elements $x,\{x, x\}$ with $\operatorname{deg} x=d-3$ as a graded commutative algebra when $d$ is odd. So with Lemma 2.2, the graded algebras in (44) have the following generators

$$
\begin{aligned}
& H_{*}\left(\Omega^{2} S^{d-1}\right) \otimes H_{*}\left(\Omega\left(S O_{d}\right)_{1}\right): x \otimes 1,\{x, x\} \otimes 1,1 \otimes \gamma_{1}, \ldots, 1 \otimes \gamma_{m-1}, 1 \otimes \gamma_{m} \\
& H_{*}\left(\Omega\left(S O_{d-1}\right)_{1}\right): f, \quad \gamma_{1}, \quad \ldots, \quad \gamma_{m-1}
\end{aligned}
$$

In the following we consider the elements in the upper horizontal line of (5) as elements in $E^{2}\left(f \mathcal{S}_{d}^{\bullet}\right)$. By comparison of the number of generators and their degree, it is plausible to expect the equation

$$
d^{2}\left(1 \otimes \gamma_{m}\right)=\{x, x\} \quad \text { up to non-zero scaler multiple. }
$$

We shall verify this equation (맘 in details. Note that there exist morphisms of spectral sequences as follows.

$$
E_{p, q}^{r}\left(\mathcal{S}_{d}^{\bullet}\right) \longrightarrow E_{p, q}^{r}\left(f \mathcal{S}_{d}^{\bullet}\right) \longleftarrow E_{p, q}^{r}\left(\underline{S O_{d-1}}{ }^{\bullet}\right) .
$$

Here the left morphism is induced by the inclusion to the unity $* \rightarrow S O_{d}$ and the right one is induced by the morphism in Lemma 2.1. We shall consider the case $r=2$. Note that $x \otimes 1,\{x, x\} \otimes 1$ come from the left hand side of the diagram (7) and $1 \otimes \gamma_{i}(1 \leq i \leq m-1)$ comes from the right hand side of the same diagram in view of the isomorphism (2) and the isomorphism $E^{2}\left({\underline{S O_{d-1}}}^{\bullet}\right) \cong H_{*}\left(\omega \mathrm{SO}_{d-1}\right) \cong H_{*}\left(\Omega\left(S O_{d-1}\right)_{1}\right)$, see also Lemma 2.2. This means these elements are cycles at any page of the middle spectral sequence in (7) as the left and right hand sides of (7) degenerate at $E^{2}$-page. $\{x, x\}$ has the odd degree and the all generators of the abutment $H_{*}\left(\Omega\left(S O_{d-1}\right)_{1}\right)$ have even degree so $\{x, x\} \otimes 1$ must be a boundary in some page. Suppose $\{x, x\} \otimes 1$ is a boundary in $E^{r}$-page but not in $E^{r-1}$-page. Take an element $y$ such that $d^{r}(y)=\{x, x\} \otimes 1$ We may write $y=y_{1}+k\left(1 \otimes \gamma_{m}\right)$ where $y_{1}$ is a polynomial of $x \otimes 1,1 \otimes \gamma_{1}, \ldots, 1 \otimes \gamma_{m-1}$, and $k$ is a scalar. But $y_{1}$ is a cycle since $1 \otimes \gamma_{1}, \ldots, 1 \otimes \gamma_{m-1}$ are cycles. This implies $d^{r}\left(y_{1}\right)=0$ and in turn, we have $k d^{r}\left(1 \otimes \bar{\gamma}_{m}\right)=$ $\{x, x\} \otimes 1$. Note that $1 \otimes \gamma_{m}$ corresponds to $1 \otimes \bar{\beta}_{m} \in 1 \otimes H_{-1,4 m-1}\left(\omega \mathrm{SO}_{d}\right)$ under the isomorphism (3) and $x$ corresponds an element $\alpha \in H_{d-1}\left(\mathcal{S}_{d}(2)\right) \subset H H_{-2, d-1}\left(H_{*}\left(\mathcal{S}_{d}\right)\right)$ and $\{x, x\}$ corresponds $\{\alpha, \alpha\} \in H_{-3,2 d-2}\left(H_{*}\left(\mathcal{S}_{d}(3)\right)\right.$ under the isomorphism (2), where the bracket $\{\alpha, \alpha\}$ denotes the algebraic Gerstenhaber bracket recalled in section 2 so the bidegree of $d^{r}$ must be $(-2,1)$, which implies $r=2$, and we have proved the equation (6) and the part 2 of the lemma for the case $\mathcal{O}=\mathcal{S}_{d}$.

For the case $\mathcal{O}=\mathcal{K}_{d}$, As the spectral sequences are natural for the inclusion $\mathcal{K}_{d} \subset \mathcal{S}_{d}$ and the image of $\{x, x\}$ by the inclusion is non-zero, which can be seen by elementary computation based on [13, Theorem 7.4], the above result for $\mathcal{O}=\mathcal{S}_{d}$ immediately implies $d^{2}\left(1 \otimes \bar{\beta}_{m}\right) \neq 0$ for $\mathcal{O}=\mathcal{K}_{d}$.

\section{An obstruction to formality and proof of Theorem 2.3}

In Lemma 2.2, we may take a generator $\beta_{m} \in H_{4 m-1}\left(S O_{d}\right)$ to be primitive. In other words, we may assume

$$
\Delta \beta_{m}=1 \otimes \beta_{m}+\beta_{m} \otimes 1
$$

where $\Delta$ denotes the Alexander-Whitney diagonal. We regard $\beta_{m}$ as an element of $H_{*}\left(f \mathcal{K}_{d}(1)\right)$ by the obvious homeomorphism $f \mathcal{K}_{d}(1)=\{i d\} \times S O_{d}=S O_{d}$. By the 
definition of a semi-direct product (see section 2) we see that the following diagram is commutative.

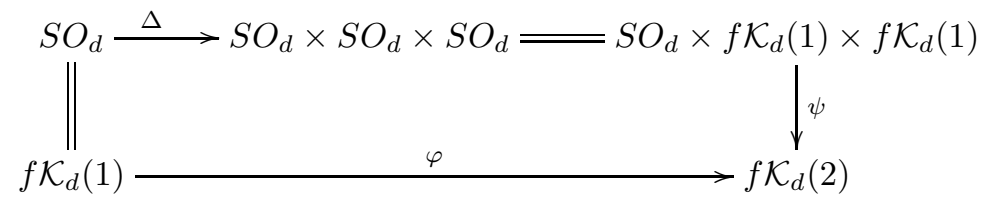

Here, $\Delta$ denotes the (topological) diagonal map, and the maps $\psi$ and $\varphi$ are given by $\psi(x, y, z)=(x \cdot \mu) \circ(y, z)$ and $\varphi(y)=y \circ_{1} \mu$ where $\mu$ is the image of the unique point by the structure map $\mathcal{A}(2) \rightarrow f \mathcal{K}_{d}(2)$. We also have an equation $\beta_{m} \cdot \mu=0$ as $H_{4 m-1}\left(\mathcal{K}_{d}(2)\right)=0$. By this equation, the equation (8), and the above diagram, we have the following equation.

$$
\beta_{m} \circ_{1} \mu=\mu \circ_{2} \beta_{m}+\mu \circ_{1} \beta_{m} \in H_{4 m-1}\left(f \mathcal{K}_{d}(2)\right) .
$$

In view of this one, we define an obstruction class for formality as follows.

Definition 4.1. Let $\mathcal{O}$ be a chain operad such that the homology $H_{*}(\mathcal{O})$ is isomorphic to $H_{*}\left(f \mathcal{K}_{d}\right)$ as a graded operad. Let $\nu \in \mathcal{O}(2)_{0}$ be a cycle which represents a generater of $H_{0}(\mathcal{O}(2)) \cong \mathbb{Q}$. Let $g \in \mathcal{O}(1)_{4 m-1}$ be a cycle. We impose the pair $(\nu, g)$ the following condition:

$$
\left[g \circ_{1} \nu\right]=\left[\nu \circ_{2} g+\nu \circ_{1} g\right] \in H_{4 m-1}(\mathcal{O}(2)) .
$$

Here, $[a]$ denotes the homology class represented by a cycle $a$.

We can pick an element $h \in \mathcal{O}(2)_{4 m}$ such that

$$
d h=\nu \circ_{2} g+\nu \circ_{1} g-g \circ_{1} \nu .
$$

As $[\nu]$ represents an associative multiplication, we can pick an element $\xi \in \mathcal{O}(3)_{1}$ such that

$$
d \xi=\nu \circ_{2} \nu-\nu \circ_{1} \nu
$$

Then, we define an obstruction cycle $\omega=\omega_{\mathcal{O}}(\nu, g) \in \mathcal{O}(3)_{4 m}$ by

$$
\begin{aligned}
\omega=\omega_{1}-\omega_{2}, & \omega_{1}=\nu \circ_{2} h-h \circ_{1} \nu+h \circ_{2} \nu-\nu \circ_{1} h \\
\omega_{2} & =g \circ_{1} \xi+\xi \circ_{1} g+\xi \circ_{2} g+\xi \circ_{3} g
\end{aligned}
$$

We will see this is a cycle for the internal differential of $\mathcal{O}(3)$ in the proof of Lemma 4.2.

As $[\nu]$ defines a structure of a multiplicative operad on $H_{*}(\mathcal{O})$, we can consider the Hochschild complex of $H_{*}(\mathcal{O})$. Its differential $\delta_{\nu}: H_{*}(\mathcal{O}(n)) \rightarrow H_{*}(\mathcal{O}(n+1))$ is given by $\delta_{\nu}([x])=\left[\nu \circ_{2} x+\sum_{i=1}^{n-1}(-1)^{n} x \circ_{i} \nu+(-1)^{n} \nu \circ_{1} x\right]$

We call the element $[\omega]$ of the quotient $H_{4 m}(\mathcal{O}(3)) / \delta_{\nu} H_{4 m}(\mathcal{O}(2))$ represented by $\omega$ the obstruction class (or set) for the pair $(\nu, g)$.

Lemma 4.2. Under the notations of Definition 4.1, $\omega$ is a cycle and the corresponding class $[\omega]$ is independent of choise of $h$ and $\xi$.

Proof. We first show $\omega$ is a cycle.

$$
\begin{aligned}
d \omega_{1} & =\nu \circ_{2} d h-d h \circ_{1} \nu+d h \circ_{2} \nu-\nu \circ_{1} d h \\
& =\nu \circ_{2}\left(\nu \circ_{2} g+\nu \circ_{1} g-g \circ_{1} \nu\right)-\left(\nu \circ_{2} g+\nu \circ_{1} g-g \circ_{1} \nu\right) \circ_{1} \nu \\
& +\left(\nu \circ_{2} g+\nu \circ_{1} g-g \circ_{1} \nu\right) \circ_{2} \nu-\nu \circ_{1}\left(\nu \circ_{2} g+\nu \circ_{1} g-g \circ_{1} \nu\right)
\end{aligned}
$$

By the associativity of partial composition, we have $\nu \circ_{2}\left(\nu \circ_{2} g\right)=\left(\nu \circ_{2} \nu\right) \circ_{3} g$ and $\left(\nu \circ_{2} g\right) \circ_{1} \nu=\left(\nu \circ_{1} \nu\right) \circ_{3} g$, for example. By using these and similar equalities, we have

$$
\begin{aligned}
d \omega_{1} & =-g \circ_{1}\left(\nu \circ_{2} \nu-\nu \circ_{1} \nu\right)+\left(\nu \circ_{2} \nu-\nu \circ_{1} \nu\right) \circ_{1} g \\
& +\left(\nu \circ_{2} \nu-\nu \circ_{1} \nu\right) \circ_{2} g+\left(\nu \circ_{2} \nu-\nu \circ_{1} \nu\right) \circ_{3} g \\
& =-g \circ_{1} d \xi+(d \xi) \circ_{1} g+(d \xi) \circ_{2} g+(d \xi) \circ_{3} g \\
& =d \omega_{2}
\end{aligned}
$$


Thus, we have $d \omega=d\left(\omega_{1}-\omega_{2}\right)=0$.

Let $h^{\prime}, h^{\prime \prime}$ (resp. $\xi^{\prime}, \xi^{\prime \prime}$ ) be two elements satisfying the condition (11) (resp. (12)) in Definition 4.1] In the rest of the proof, $\omega^{\prime}=\omega_{1}^{\prime}-\omega_{2}^{\prime}$ and $\omega^{\prime \prime}=\omega_{1}^{\prime \prime}-\omega_{2}^{\prime \prime}$ denote the elements defined by the equation (13), using $\left(h^{\prime}, \xi^{\prime}\right)$ and $\left(h^{\prime \prime}, \xi^{\prime \prime}\right)$ respectively. As $h^{\prime}-h^{\prime \prime}$ is a cycle, the class of $\omega_{1}^{\prime}-\omega_{1}^{\prime \prime}$ belongs to $\delta_{\nu} H_{4 m}(\mathcal{O}(2))$. As $H_{1}(\mathcal{O}(3)) \cong H_{1}\left(f \mathcal{K}_{d}(3)\right)=0$ and $\xi^{\prime}-\xi^{\prime \prime} \in \mathcal{O}(3)_{1}$ is a cycle, it is a boundary. So $\omega_{2}^{\prime}-\omega_{2}^{\prime \prime}$ is also boundary. Thus $\omega^{\prime}-\omega^{\prime \prime}$ represents the zero class in $H_{4 m}(\mathcal{O}(3)) / \delta_{\nu} H_{4 m}(\mathcal{O}(2))$. In other words, $\left[\omega^{\prime}\right]=\left[\omega^{\prime \prime}\right]$.

The class $[\omega]$ may depend not only on the classes $[\nu]$ and $[g]$ but also on the cycles $\nu$ and $g$. We must take care about these choices in the proof of Theorem 2.3 .

Remark 4.3. Note that the equation (10) is equivalent to the equation $\{[g],[\nu]\}=0$, where $\{-,-\}$ denotes the Gerstenhaber bracket on the Hochschild homology of $H_{*}(\mathcal{O})$. and the associativity equation $[\nu] \circ_{2}[\nu]-[\nu] \circ_{1}[\nu]=0$ is equivalent to the equation $\frac{1}{2}\{[\nu],[\nu]\}=0$. Our class $[\omega]$ is something like "Massey triple bracket" for $[g],[\nu],[\nu]$. This point of view was pointed out to the author by V. Turchin. The cycle $\omega$ should be defined as $\{\nu, h\} \pm\{g, \xi\}$ under the notations of Definition 4.1 if the Hochschild complex of $\mathcal{O}$ were a differential graded Lie algebra (with some degree shift). But actually it is not so because $\{-,-\}$ is not (anti-)derivative for the internal differential of $\mathcal{O}$, and the actual definition is different from the above formula in signs. This makes it difficult to prove the class $[\omega]$ does not depend on choices of cycles $g, \nu$ in each class.

We use a model category of chain operads. For general theory of model categories, see [6]. It is known that the category of chain operads has a model category structure where weak equivalences are the same as those given in section 2] (see [27, Theorem 2.1] or [16, Theorem 1.1], see also [5] for a model category of symmetric operads).

Proof of Theorem 2.3. We shall prove part 1. The proof of part 2 is completely analogous and we omit it.

Set $\mathcal{O}=C_{*}\left(f \mathcal{K}_{d}\right)$. Let $\nu \in \mathcal{O}(2)$ be the 0 -cycle represented by the image of the unique point by the structure morphism $\mathcal{A} \rightarrow f \mathcal{K}_{d}$ and $g \in \mathcal{O}(1)_{4 m-1}$ be any cycle which represents the primitive generator $\beta_{m}$. The pair $(\nu, g)$ satisfies the condition (10) in Definition 4.1. As $\nu$ is strictly associative, we may take zero as $\xi$. In this case, by definition, $E^{2}\left(f \mathcal{O}^{\bullet}\right)$ is naturally considered as a sub-vector space of $H_{*}(\mathcal{O}(-)) / \delta_{\nu} H_{*}(\mathcal{O}(-))$. Under this identification, we easily see $[\omega(\nu, g)]=d_{2}\left(1 \otimes \bar{\beta}_{4 m}\right)$ by unwinding the definition of the differential of the spectral sequence. So by the part 2 of Lemma 3.1. we see $[\omega] \in$ $H_{4 m}(\mathcal{O}(3)) / \delta_{\nu} H_{4 m}(\mathcal{O}(2))$ is non-zero for this choise of $\nu$ and $g$. Let $\mathcal{A}_{\infty}$ be the Stasheff's associahedral chain operad. We consider the non-unital version i.e., $\mathcal{A}_{\infty}(0)=0$ so $\mathcal{A}_{\infty}$ is a cofibrant operad and has a set of generators $\left\{\nu_{i} \mid i \geq 1\right\}$ consisting of the fundamental classes of cells of the associahedra (see [2, 20]). We define a morphism of operads $f: \mathcal{A}_{\infty} \rightarrow$ $\mathcal{O}$ by $\nu_{1} \mapsto \nu$, and taking the other generators to zeros. By a functorial factorization, $f$ is factorized as $\mathcal{A}_{\infty} \underset{i}{\longrightarrow} \mathcal{P} \underset{p}{\stackrel{\sim}{\sim}} \mathcal{O}$. As $\mathcal{A}_{\infty}$ is cofibrant, so is $\mathcal{P}$.

Set $\mathcal{H}=H_{*}(\mathcal{O})$. Suppose $\mathcal{O}$ is formal. In other words, $\mathcal{O}$ and $\mathcal{H}$ are connected by a chain of weak equialences of operads. As $\mathcal{P}$ is cofibrant (and any chain operad is fibrant), by the theory of model categories, there exists a weak equivalence $q: \mathcal{P} \rightarrow \mathcal{H}$. We can take a cycle $g^{\prime} \in \mathcal{P}(1)$ such that the class $\left[g^{\prime}\right]$ goes to $\beta_{m}$ by $p_{*}$. The pair $\left(i\left(\nu_{1}\right), g^{\prime}\right)$ satisfies the condition of Definition 4.1 for $\mathcal{P}$. It is clear that the pairs $\left(\nu, p\left(g^{\prime}\right)\right),\left(q\left(g^{\prime}\right), q i\left(\nu_{1}\right)\right)$ also 
satisfy the condition in Definition 4.1. We have isomorphisms

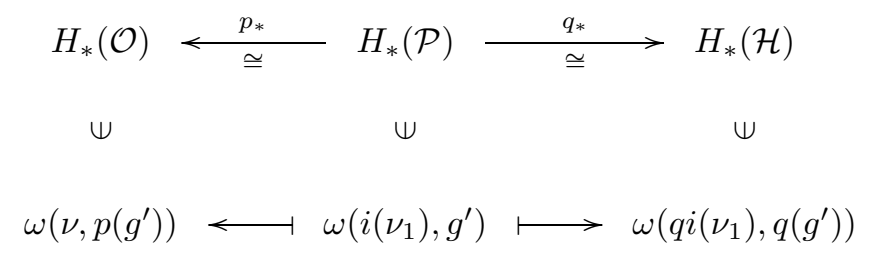

By these isomorphisms, $\left[\omega\left(\nu, p\left(g^{\prime}\right)\right)\right]$ corresponds to $\left[\omega\left(q i\left(\nu_{1}\right), q\left(g^{\prime}\right)\right)\right]$. As we show in the above, $\left[\omega\left(\nu, p\left(g^{\prime}\right)\right)\right]$ is non-zero. On the other hand, the differential of $\mathcal{H}$ is zero, we may choose zeros as $h$ and $\xi$ in the definition of $\omega\left(q i\left(\nu_{1}\right), q\left(g^{\prime}\right)\right)$. Hence $\omega\left(q i\left(\nu_{1}\right), q\left(g^{\prime}\right)\right)$ represents zero in $H_{4 m}(\mathcal{H}(3)) / \delta_{q\left(\nu_{1}\right)} H_{4 m}(\mathcal{H}(2))$. This is a contradiction.

\section{ACKNOWLEDGEMENTS}

The author is grateful to Thomas Goodwillie, Robin Koytcheff, Dev Prakash Sinha, Paul Arnaud Songhafouo Tsopméné, and Victor Turchin for very valuable discussions and comments about the subject of this paper. He deeply thanks Dev Sinha and Ismar Volić for giving me an opportunity to meet the above people and taking care of my trip kindly. He is also grateful to Masana Harada for fruitful comments for this paper and refreshing conversation.

\section{REFERENCES}

[1] A. K. Bousfield, On the homology spectral sequence of a cosimplicial space, Amer. J. Math. 109 (1987), no. 2, 361-394.

[2] J. D. Stasheff, Homotopy associativity of H-spaces. II Trans. Amer. Math. Soc. 108 (1963), no.2, 293-312.

[3] M. Gerstenhaber, A. A. Voronov, Homotopy G-algebras and moduli space operad, Internat. Math. Res. Notices (1995), no. 3, 141-153.

[4] I.Kriz J.P.May, Operads, Algebras, Modules and motives Astérisque, 233 (1995), iv+145pp.

[5] V. Hinich, Homological algebra of homotopy algebras. Comm. Algebra, 25 (1997), no. 10, 3291-3323.

[6] M. Hovey, Model categories, Mathematical Surveys and Monographs, 63 (1999), American Mathematical Society, Providence, RI, xii+209 pp.

[7] M. Kontsevich, Operads and motives in deformation quantization, Moshé Flato (1937-1998). Lett. Math. Phys. 48 (1999), no. 1, 35-72.

[8] P. Salvatore, N. Wahl, Framed discs operads and Batalin-Vilkovisky algebras, Q. J. Math. 54 (2003), no. 2, 213-231.

[9] D. E. Tamarkin, Formality of chain operad of little discs, Lett. Math. Phys. 66 (2003), no. 1-2, 65-72.

[10] J. E. McClure, J. H. Smith, Cosimplicial objects and little $n$-cubes. I, Amer. J. Math. 126 (2004), no. $5,1109-1153$.

[11] V. Turchin (Tourtchine), On the homology of the spaces of long knots, Advances in Topological Quantum Field Theory, NATO Sci. Ser. II Math. Phys. Chem. 179 (2004), Kluwer Acad. Publ., Dordrecht, 23-52.

[12] P. Salvatore, Knots, operads, and double loop spaces, Int. Math. Res. Not. (2006), Art. ID 13628, 22 pp.

[13] Dev. P. Sinha, Operads and knot spaces, J. Amer. Math. Soc. 19 (2006), no. 2, 461-486.

[14] K. Sakai, Poisson structures on the homology of the space of knots, Groups, homotopy and configuration spaces, Geom. Topol. Monogr. 13 (2008), 463-482.

[15] P. Lambrechts, V. Turchin and I. Volić, The rational homology of spaces of long knots in codimension $>$ 2, Geom. Topol. 14 (2010), no. 4, 2151-2187.

[16] F. Muro, Homotopy theory of nonsymmetric operads, Algebr. Geom. Topol. 11 (2011), no. 3, 15411599.

[17] J. Giansiracusa and P. Salvatore, Cyclic operad formality for compactified moduli spaces of genus zero surfaces . Trans. Amer .Math. Soc. 364 (2012), 5881-5911

[18] P. Boavida de Brito, M. Weiss, Manifold calculus and homotopy sheaves, Homology Homotopy Appl. 15 (2013), no. 2, 361-383.

[19] V. Turchin, Context-free manifold calculus and the Fulton-MacPherson operad, Algebr. Geom. Topol. 13 (2013), no. 3, 1243-1271.

[20] F. Muro, and A. Tonks, Unital associahedra, Forum Math. 26 (2014), no. 2, 593-620. 
[21] G. Arone, P. Lambrechts, and I. Volić, Calculus of functors, operad formality, and rational homology of embedding spaces, Acta Math. 199 (2007), no. 2, 153-198.

[22] P. A. Songhafouo Tsopméné, Formality of Sinhafs cosimplicial model for long knots spaces and the Gerstenhaber algebra structure of homology, Algebr. Geom. Topol. 13 (2013), no.4, 2193-2205.

[23] P. Lambrechts, I. Volić, Formality of the little $N$-disks operad, Mem. Amer. Math. Soc. 230 No. 1079. American Mathematical Society, 2014.

[24] G. Arone, V. Turchin, On the rational homology of high-dimensional analogues of spaces of long knots, Geom. Topol. 18 (2014), no. 3, 1261-1322.

[25] V. Turchin, T. Willwacher, Relative (non-)formality of the little cubes operads and the algebraic Cerf Lemma, preprint, (2014), arXiv:1409.0163

[26] M. Livernet, Non-formality of the Swiss-cheese operad, J. Topol. 8 (2015), no.4, 1156-1166.

[27] S.Moriya, Multiplicative formality of operads and Sinha's spectral sequence for long knots, Kyoto. J. Math. 55 (2015), no. 1, 17-27.

[28] P. A. Songhafouo Tsopméné, The rational homology of spaces of long links, Algebr. Geom. Topol. 16 (2016), no. 2, 757-782.

Department of Mathematics and Information Sciences, Osaka Prefecture University, Sakai, 599-8531, JAPAN

E-mail address: moriyasy@gmail.com 